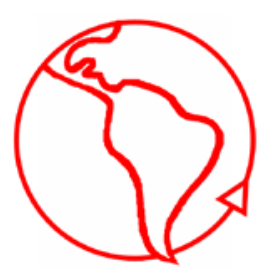

\title{
La economía de la sociedad como sistema autopoiético
}

\author{
The Economy of Society as an Autopoietic System
}

Niklas Luhmann

Facultad de Sociología, Universidad de Bielefeld, Alemania

Resumen

Toda sociedad tiene que resolver problemas económicos. En la sociedad moderna esto sucede con la ayuda de un sistema económico diferenciado. A pesar de todas sus interdependencias y vínculos causales, los cuales se incrementan aún más debido a la diferenciación funcional de la sociedad, el sistema económico opera como un subsistema "autopoiético" bajo autonomía funcional. Este sistema se encuentra cerrado por su circularidad recursiva. Está compuesto de pagos que son posibles en base a otros pagos y que permiten pagos adicionales. En este sentido, los elementos del sistema son producidos por los elementos del sistema y el entorno del sistema no contiene ningún elemento de este tipo. El sistema económico es al mismo tiempo un sistema abierto, ya que sus operaciones están adaptadas a las necesidades de su entorno social y humano, y debido a que su función consiste en garantizar la perspectiva que ulteriores demandas serán satisfechas en un futuro cercano. En este sentido la clausura del sistema es condición de su apertura, y viceversa. El presente artículo traza algunas consecuencias de este enfoque teórico.

Palabras Clave: Teoría de sistemas sociales, Diferenciación Funcional; Economía; Autopoiesis, Autorreferencia

\begin{abstract}
Every society has to solve economic problems. In modern society this occurs with the help of a differentiated economic system. In spite of all its interdependencies and causal links, which tend to increase due to functional differentiation of society, economic system operates as an "autopoietic" subsystem under functional autonomy. This system is closed by its recursive circularity. It consists of payments that are based on other payments and that allow further payments. In this sense, the elements of the system are produced by the elements of the system and the environment of the system contains no elements of that type. Economic system is at the same time an open system since its operations are adapted to the needs of its social and human environment, and because its function consists of ensuring the prospect that future demands will be satisfied in a nearby future. In this sense the closeness of the system is the condition for its openness and vice versa. This article outlines some consequences of this theoretical approach.
\end{abstract}

Keywords: Social Systems Theory; Functional Differentiation; Economy; Autopoiesis; Self- Reference

$I^{*}$

Sin duda la transición a la economía monetaria ha desempeñado un importante rol en el surgimiento de la sociedad moderna, algunos incluso sostienen que ha jugado el papel

\footnotetext{
* Traducido por Hugo Cadenas. Con autorización de: Die Zeitschrift für Soziologie, Bielefeld, Alemania. Título original: Die Wirtschaft der Gesellschaft als autopoietisches System. Zeitschrift für Soziologie, Jg. 13, Heft 4, Oktober 1984, S. 308327. El traductor agradece las observaciones y comentarios por parte de Marcelo Arnold y Anahí Urquiza.
} 
decisivo. En efecto, lo característico de la sociedad moderna es a menudo expresado en conceptos que suponen la economía monetaria. Solo se pude discutir con el tópico relativo a si estamos hablando de capitalismo 0 de sociedad industrial ${ }^{1}$ utilizando diferentes versiones de este punto de vista, es decir solamente en lo relativo a la constitución económica. Que en el fondo se trata de la sociedad, queda como un supuesto. En lo sucesivo no nos ocuparemos de la discusión entre capitalismo o sociedad industrial sino de aquello que queda como supuesto. No intentamos acá hacer una contribución sobre la génesis de la sociedad moderna, sino más bien aclarar una pregunta previa al respecto.

En este sentido, se trata de la economía y la sociedad. Sin embargo esta fórmula no nos conduce al problema de fondo. Más bien lleva a un engaño, pues invita a representar a la economía y a la sociedad cómo dos hechos independientes que deben estar relacionados entre sí. Sin embargo la tradición sociológica nunca ha pensado realmente de este modo ni en la economía política ni tampoco en su vertiente sociológica. Más bien, a partir del juego conceptual de Hegel, ha ocurrido lo contrario: se compara economía y sociedad como si la sociedad estuviera, por así decir, infectada por la economía, para luego posteriormente depositar las esperanzas de su salvación de diversas maneras: por medio un Estado fundado en una ética, a través de la abolición de las clases sociales, por una nueva forma de solidaridad social, etc. Esta postura pudo enlazar con una tradición crítica hacia el dinero, con una animadversión hacia la prostitución, la venalidad, el fetichismo de las mercancías, el capitalismo o la plutocracia, y pudo así compensar los vacíos de su teoría mediante tal animosidad.

Sin duda, esta tradición se basó en ocasiones en un formidable esfuerzo teórico. Particularmente los clásicos de la economía política, desde Adam Smith hasta Karl Marx, vuelven hoy en día a ser tomados en cuenta. Las siguientes reflexiones se fundamentan, sin embargo, de un modo totalmente distinto, pues la teoría clásica surgió como reacción a la históricamente nueva diferenciación del sistema económico basado en el dinero. Esta peculiaridad, sin embargo, no pudo ser comprendida de inmediato y de manera adecuada en dicha tradición ${ }^{2}$.

Para lograr el distanciamiento de esta tradición debiesen bastar solamente algunas precisiones. Lo que Ilamó poderosamente la atención con la naciente industrialización fue sobre todo el aumento de la productividad. La teoría de la economía fue concebida por este motivo como una teoría de la producción económica. La antigua idea de una cantidad de bienes limitados naturalmente -que habían actualizado posteriormente los fisiócratas- , en la cual el aumento de la prosperidad era atribuido exclusivamente al factor agrícola, se hizo luego evidentemente insostenible. Precisamente por esto el trabajo fue valorado como el más importante factor de la producción -si es que no el único- que permitía explicar dicho aumento ${ }^{3}$. La recursividad circularmente cerrada del sistema fue expresada en la teoría de los factores de la producción y no en la teoría del dinero. Esto significó que solamente se tomaron en cuenta estos factores, cuya disponibilidad y renovación podrían a su vez ser considerados como producción, es decir que costaban trabajo o dinero. En última instancia, todo volvía al trabajo. La división del trabajo fue considerada el "milagro" del aumento de la eficiencia y para hacerla posible era necesaria la división del dinero. La diferencia entre estado de naturaleza/ civilización fue reinterpretada como la diferencia entre sociedad con división del trabajo/sociedad sin división del trabajo (i. e. Torrens 1821) y de acuerdo con esto la preocupante discrepancia entre riqueza y pobreza debió

\footnotetext{
${ }^{1}$ Así está planteado el tema central del Decimosexto Congreso Alemán de Sociología celebrado en Frankfurt en 1968. Ver Adorno (1969).

${ }^{2}$ La razón de porqué esto no fue inmediatamente posible se debe a problemas de las teorías de reflexión inmanentemente sistémicas, sobre las cuales volveremos en el capítulo VIII.

${ }^{3}$ Los fisiócratas todavía veían al trabajo bajo una premisa de suma constante, ya que este consume las fuerzas que son necesarias para su propia producción. Véase, por ejemplo Schlettwein (1779, Vol. I: 72 y ss.).
} 
ser aceptada como condición de la división del trabajo ${ }^{4}$. Incluso el concepto de capital fue reducido a producción, definido por ejemplo como un medio de producción producido. El dinero fue celebrado naturalmente como un requisito indispensable de la economía de mercado y como un logro civilizatorio, sin embargo este no fue emplazado en dicha teoría en el lugar al que pertenece 5 . Fue concebido por Adam Smith como un instrumento de distribución, y la distribución es por supuesto secundaria a la producción. Con esto se produjo una contradicción con el concepto de trabajo, el que por un lado sustenta toda la construcción teórica y por otro lado es considerado solamente como un colaborador de la producción de mercancías (orientadas hacia el dinero) ${ }^{6}$. Asimismo se produjeron contradicciones con el concepto de producción, específicamente con el concepto de producción no productiva (incapaz de ser vendida) (Hodgskin 1827: 50 y s.) y con la afirmación de la simultánea subproducción y sobreproducción (Hodgskin 1827: 245 y s., 264). La teoría económica tuvo que reaccionar luego a esta centralización en torno al problema de la distribución.

La teoría del materialismo dialéctico se bifurca desde acá. Esta teoría detecta los problemas derivados de la economía monetaria de manera mucho más aguda que antes ${ }^{7}$. Su mayor hazaña fue sobre todo haber develado que su teoría de las contradicciones se corresponde con contradicciones de la realidad social, es decir ellas se entienden como un momento del objeto de su teoría. Esta teoría explica la inestabilidad social debido a contradicciones lógicas (algo que perfectamente puede ser puesto en duda) ${ }^{8}$ y motiva expectativas de síntesis y sus respectivos esfuerzos, sin haber sido aclarada adecuadamente la función del dinero en el contexto de la economía y la sociedad.

Ciertamente, no se pudo saber de antemano que justamente este defecto resultaría fundamental y que precisamente la teoría del dinero (y no una teoría de las necesidades, de las relaciones de producción o de la división del trabajo) es quien conduce a una "mejor" comprensión de la relación entre economía y sociedad. Aunque es cierto que la teoría clásica considera una situación en la que ya no se aplica el principio del volumen constante de bienes, y que por esto el trabajo es considerado de importancia fundamental, resulta claro que cualquier revisión teórica que comience desde aquí puede parecer revolucionaria.

Las siguientes reflexiones tienen por objetivo reemplazar al factor trabajo (en la ubicación teórica antes mencionada) por el concepto de codificación de la comunicación. Se puede entender al dinero como codificación de las operaciones económicas y a esta codificación como duplicación de la escasez. Hay, pues, dos lenguajes de la escasez: el de los bienes y el del dinero, los cuales demandan diferentes condiciones. En la economía moderna se llevan a cabo todas las operaciones económicas, es decir ambos lenguajes de la escasez emplean de manera simultánea el código de la economía -y solo a este código- para

\footnotetext{
${ }^{4}$ Así, incluso antes de Adam Smith, por ejemplo Boesnier de l'Orme (1775: 57 y ss.). Más tarde el argumento se desplazó más bien hacia los "efectos" de la división del trabajo y su respectiva abstracción de necesidades. Véase Hegel (1983: 193 y ss.).

${ }^{5}$ Torrens, por ejemplo, observa en el desarrollo de su teoría de la economía política en un primer momento al dinero bajo el fundamento que: "cuando las hipótesis que empleamos con el propósito de trazar y elucidar los principios de la ciencia económica tienen que ver con el dinero nos vemos propensos a caer en la confusión y el error, debido a que nuestra atención está dirigida no a aquello que es esencial e inherente al caso que tenemos en frente, sino a alguna circunstancia o accidente conectado con la mercancía que es empleada como medio de intercambio y medida práctica de valor" (1821: 290). De manera similar en Hodgskin (1827: 179).

${ }^{6}$ Ver Hodgskin (1827: 28) respecto de un lado de esto y (1827: 50) irespecto del lado contrario!

${ }^{7}$ Esto sin embargo puede ser puesto duda. Ver Luhmann (1983c) o también Hodgskin (1827): “El campesino que produce mucho maíz, cuyo amo se arruinó por su reducido precio, no tiene qué comer y con qué cubrirse. El tejedor que provee al mundo con ropas, cuyo amo emprendió peligrosas aventuras para tentar a los salvajes a utilizar sus productos, se va desgastando con el hambre y la desnudez en medio de una temporada inclemente".

${ }^{8}$ Véase, en general Elster (1981).
} 
pagar por servicios. La estructura de la economía consiste en el condicionamiento de este entramado operacional. Solo se puede reducir la escasez de bienes debido a que junto a esta se ubica al mismo tiempo una segunda escasez, a saber, una escasez de amortiguación [Auffangknappheit]. Esta codificación contempla evidentemente al trabajo, no obstante la codificación en sí es la razón estructural para el éxito y no el trabajo como tal, el que por su parte es imposible de concebir como un factor independiente en el sistema cerrado de la economía del dinero.

Si se buscan puntos de apoyo en la sociología teórica para esta idea, uno se encuentra con la sugerencia de Parsons de entender al dinero como un medio de intercambio simbólicamente generalizado ${ }^{9}$ en el contexto de una teoría de la diferenciación funcional. Este punto de partida sin embargo está ligado al diseño teórico parsoniano de las tablas de referencias cruzadas, el cual no ha evolucionado de manera significativa. Algunas expectativas ven aquí con esperanzas una teoría sucesora de los problemas planteados por Max Weber sobre el destino de la racionalidad occidental (Baum 1976, 1976a), otros sostienen que esta teoría en realidad solo funciona respecto del tema del dinero, aunque también posiblemente en el área del poder político y administrativo, ya que como teoría general solo lograría expresar una sobreestimación de la importancia de la racionalidad sistémico-técnica para la sociedad moderna ${ }^{10}$. Mi impresión es que los medios de intercambio simbólicamente generalizados constituyeron un importante elemento teórico que quedó en un estado de subdesarrollo, el cual sin embargo debiera permanecer, por así decir, en situación de espera hasta que la teoría de sistemas en general, y la teoría social en particular, hayan alcanzado un nivel de avance adecuado.

Las siguientes consideraciones tienen por objetivo establecer las conexiones actualmente posibles. Ellas se desligan del concepto de sistema del marco teórico parsoniano y en su lugar recurren a una teoría general de sistemas autorreferenciales. Dicha teoría puede comprender de manera más precisa: (1) qué problemas se deben resolver en el curso de la diferenciación de la sociedad y (2) cómo el medio de comunicación dinero contribuye específicamente a resolver estos problemas. Dicho de otro modo, se mantiene el supuesto relativo a una relación entre diferenciación funcional y desarrollo de los medios, incluyendo el desarrollo del dinero, pero sus fundamentos son reemplazados como consecuencia de un cambio de paradigma en la teoría general de sistemas.

\section{II}

La teoría de sistemas de las dos últimas décadas ha empezado a recoger e incorporar problemas de autorreferencialidad. Inicialmente aparecieron en primer plano problemas de reflexión relativos a la identidad del sistema en el sistema y también problemas de autonomía o autorganización -los primeros relacionados más bien con sistemas psíquicos y los segundos con máquinas que procesan información y con sistemas vivos. El objeto de la autorreferencia fue solamente la unidad del sistema o estructura presentada como un objeto $y$, especialmente, el cambio en la estructura del sistema. Desde entonces este enfoque teórico se ha radicalizado. Teniendo en cuenta los avances de la investigación en áreas subatómicas y sub-subatómicas, la teoría debe adecuarse a la posibilidad que el mundo se amplíe hacia abajo y se haga igualmente infinito, tanto en lo pequeño como en lo grande. No existen pues elementos finales indisolubles de los cuales los sistemas estén "compuestos" y el orden no puede entenderse simplemente como una red de relaciones entre elementos. Se debe dar paso a las teorías que entienden a todo lo que actúa como unidad en el sistema como un rendimiento propio del sistema. También aquellas unidades elementales (las que no se pueden disolver adicionalmente en el sistema) obtienen su unidad por el propio sistema y solo en el contexto funcional de este. Cada elemento es siempre una reducción de complejidad subyacente en el contexto funcional del sistema, la

\footnotetext{
${ }^{9}$ Respecto de las contribuciones más importantes al respecto, véase: Parsons (1980).

${ }^{10}$ Como sostiene especialmente Habermas (1980, 1981, Bd. 2: 384 y ss., 470 y ss.)
} 
que es tratada como unidad en el sistema y por tanto tiene capacidad de conexión. Los sistemas producen los elementos de los que están hechos, a través de los propios elementos que los componen. Para explicar esta situación se ha introducido el concepto de sistema autopoiético propuesto por Maturana ${ }^{11}$.

Los sistemas autopoiéticos son sistemas cerrados en el sentido que lo que emplean como unidad para su propia reproducción (es decir: sus elementos, sus procesos, su sí mismo) no se puede obtener de su entorno. Sin embargo son también sistemas abiertos en el sentido que solo pueden realizar su autorreproducción en un entorno, en una diferencia con un entorno. Por tanto, clausura y apertura ya no pueden ser entendidas como una diferencia entre tipos. Se trata de una relación de combinaciones, de una relación de aumentos, o del resultado combinatorio de una morfogénesis evolutiva.

No resulta difícil esbozar una teoría de la sociedad a partir de esta idea teórica. La sociedad es un sistema autopoiético basado en comunicación con sentido. Se compone de comunicaciones, solamente de comunicaciones y de todas las comunicaciones. Reproduce comunicación a través de comunicación. Lo que sea que ocurra como comunicación es por tanto ejecución y al mismo tiempo reproducción de la sociedad. No puede haber entonces comunicación con el entorno ni en el entorno de la sociedad. En este sentido es el sistema de comunicación sociedad un sistema cerrado. Sin embargo este solamente es posible en un entorno, en especial gracias a la conciencia psíquica, a la vida orgánica, a materializaciones físicas, a la evolución de soles y átomos. La sociedad registra esta situación estableciéndose a sí misma como un sistema abierto. Ella comunica sobre algo sobre temas que conciernen a su entorno, a sí misma o a la comunicación que se ejecuta en el momento. La sociedad es por tanto un sistema abierto y cerrado al mismo tiempo, y la comunicación es la forma de su operación elemental, la que ejecuta constantemente esta combinación y la reproduce.

$\mathrm{Si}$ aceptamos este enfoque teórico para el sistema de la sociedad salta a la vista un problema adicional. Nos referimos a la diferenciación del sistema de la sociedad. Solo la sociedad puede ser entendida como un sistema comunicacionalmente cerrado. Solo ella integra todas las comunicaciones. Solo ella obtiene su propia unidad como autopoiesis de la comunicación. Para los demás subsistemas de la sociedad no aplica lo mismo, pues ellos operan en un entorno intrasocial donde hay asimismo comunicaciones. Los subsistemas se comunican así con sus entornos (y no solo sobre sus entornos). La economía, por ejemplo, paga impuestos. Es cierto que todos los subsistemas utilizan la comunicación como un modus propio para sus operaciones. Ellos constan también solamente de comunicaciones y son por esto subsistemas de la sociedad, son coejecutores de la reproducción de la sociedad. Sin embargo no pueden clausurarse por medio de la comunicación, no pueden distinguirse de su entorno como sistema de comunicación. Para poder constituirse en sistemas autopoiéticos, necesitan de un principio de constitución de unidad válido solamente para ellos mismos, para el que no exista un equivalente en su entorno.

No es necesario limitar el concepto de diferenciación de la sociedad exclusivamente a la formación de subsistemas autopoiéticos. Hay subsistemas de la sociedad que si bien forman su propia identidad y sus límites sistémicos y se orientan a sí mismos, no logran alcanzar exclusividad y clausura recursiva en sus operaciones elementales. Piénsese solamente en la relación entre familia y escuela, y adicionalmente en la relación de ambas con su entorno dentro de la sociedad, donde puede perfectamente existir algo similar a la socialización y puede haber incluso socialización intencional (como por ejemplo en el caso de los aprendices en las empresas o el Partido Verde alemán en el parlamento). Pero puede haber también subsistemas autopoiéticos de la sociedad, como queremos mostrar

\footnotetext{
${ }^{11}$ Véase la traducción alemana de Maturana (1982).
} 
para el caso de la economía. La intuición detrás es que en estos casos se alcanza un protagonismo social particular, precisamente porque bajo la forma de la clausura autorreferencial se evaden con más fuerza las influencias del entorno social. Además intuimos que la autopoiesis en el plano de los subsistemas está relacionada con, o mejor dicho condicionada por, la diferenciación de determinados medios de comunicación simbólicamente generalizados.

Formulado a la hechura de la sociología empírica, se trata de una relación de:

1) Forma y proporción (grado de autonomización) de la diferenciación sistémica de la sociedad;

2) diferenciación de códigos especiales para medios de comunicación simbólicamente generalizados;

3) formas de combinación entre clausura (autopoiesis) y apertura en el plano de los subsistemas de la sociedad y sus operaciones elementales; e

4) importancia relativa de los subsistemas sociales formados de esta manera (independientemente de cualquier "primacía" lógica o natural de determinadas funciones).

Con esto nos referimos a un entramado demostrable empíricamente (el cual sin embargo es inaccesible sin una orientación teórica considerable) que, como resultado de la evolución socio-cultural (es decir, sin una necesidad inmanente), define la tipicidad estructural de la sociedad moderna.

\section{III}

La economía adquiere su unidad como sistema autopoiético, producido y reproducido por sí mismo, mediante el empleo de su propio tipo de elementos, los cuales sola y exclusivamente aparecen en la economía, es decir obtienen su unidad solo en referencia recursiva a otros elementos del propio sistema. El unit-act de la economía lo constituyen los pagos. Los pagos poseen todas las propiedades de un elemento autopoiético: solo son posibles sobre la base de otros pagos y no tienen otro propósito que el de permitir pagos en la relación recursiva de la autopoiesis de la economía. La definición de autopoiesis acuñada en referencia, en primer lugar, a sistemas vivos (especialmente células) se acomoda a este caso: "La organización autopoiética se define como una unidad realizada por una red de producciones de componentes ${ }^{12}$, los cuales: 1) participan recursivamente en la reconstrucción de la misma red de producción de componentes que los produjo; y, 2) realizan la red de producciones como una unidad en el espacio en el que los componentes existen." (Maturana 1982: 158) ${ }^{13}$. Adicionalmente, la aplicación de esta teoría a la sociedad y sus subsistemas está basada totalmente en elementos temporales, es decir, en acontecimientos que desaparecen a medida que surgen. La economía se compone de incesantes nuevos pagos. Si los pagos dejasen de ocurrir, la economía simplemente dejaría de existir como un sistema diferenciado. Sus eventos basales están bajo presión de una continua autorrenovación, y esta es justamente la razón de su clausura recursiva. Para poder llegar a ser una unidad elemental, un pago debe referirse a

\footnotetext{
${ }^{12}$ Se observa particularmente que no se define por un observador (científicamente equipado), isino por sí mismo!

${ }^{13}$ En la versión original en inglés se habla de componentes [components]. Nosotros hemos hablado de "elementos" con el fin de distinguir terminológicamente con claridad entre elemento y subsistema. Otra definición con ligeras variaciones incluye con más claridad el requisito de formación de fronteras: "Sostenemos que hay sistemas que son definidos como unidades como redes de producción de componentes que (1) recursivamente, a través de sus interacciones, generan y realizan la red que los produce, y (2) constituyen, en el espacio en el que existen, los límites de esta red como componentes que participan en la realización de esta red" (Maturana 1981: 217).
} 
otros pagos. Ellos constituyen eventos en el sentido exacto de la cosmología de Alfred North Whitehead: una unidad de self-identity y self-diversity ${ }^{14}$.

La tesis relativa a que el sistema económico se compone de pagos está formulada de manera demasiado simple en un aspecto importante. Vamos a dejar esto así por razones de simplificación, pero se debe indicar todavía una complicación adicional: no se trata solamente de los pagos, sino también de los no- pagos. La decisión de no comprar coches nuevos porque estos son demasiado caros, es también con certeza un hecho natural en el sistema económico; y esto incluye asimismo a la situación de alguien que se queda en la mera abstención, sin tener otros planes para la suma de dinero que posee. Se debe sin embargo favorecer -y la delimitación presenta dificultades, como sabe cualquiera luego de una larga reflexión acerca de sus propias abstenciones- que el pago se produzca como un deseo, como expectativa, como una obligación que de alguna manera estaba cercana y sin embargo no se había actualizado. Los pagos y no-pagos surgen mediante un esquematismo de eventos acoplados, uno implica siempre la negación del otro. Quien paga no puede simplemente retener su propio dinero, y quien sí lo retiene no puede pagar, por lo que una orientación compartida se mueve paralelamente siempre en sentido contrario. Una vez obtenido el dinero, se tiene la libertad para gastarlo o retenerlo; las posibilidades se complementan, por así decir, a través de la decisión del otro. La decisión de renunciar a la libertad de elegir consiste precisamente en entregar dicha libertad a otros, y solo se puede obtener esta libertad a través de dicha renuncia - como también sucede en el proceso general de comunicación, donde quien comunica se compromete abriendo a los demás la oportunidad de aceptar o rechazar sus propuestas de sentido. En este sentido el dinero "circula". Expresado de una manera más precisa, se tendría que decir que la autorreferencia del sistema está mediada a nivel de sus operaciones basales por la -a veces requerida y a veces obligada- negación decisionalmente compartida de su contrario. Desde la teoría de sistemas se debe destacar que precisamente esta duplicación de la operación elemental (que niega los pagos con no- pagos y los no-pagos con pagos) diferencia al sistema, pues en el entorno de la economía no existen tales correspondencias. La idea orientada al intercambio o compra de "mercancías por dinero", según la cual la circulación de las mercancías se dirige en dirección opuesta a la circulación del dinero conduce a engaño. En el lado del dinero la operación pago/no- pago está condicionada (o es condicionable) de una manera completamente diferente que en el lado de las mercancías.

El sistema no puede nunca estar en equilibrio ${ }^{15}$. Él efectúa su autopoiesis solamente a través de pagos, con ayuda ciertamente de aquel esquematismo binario que fuerza a pagar, así como a no pagar, mediante la presión de negación de la autorreferencia. Nada sucede de manera simple en la economía. Cada operación adquiere su unidad como elemento del sistema relacionándose con otros elementos del sistema a través de la negación de su opuesto, y esta posibilidad en sí misma es un resultado de las operaciones elementales del sistema. Una comprensión de la economía que ubica a los pagos como operaciones básicas del sistema, puede luego tratar a todo aquello que sirve como concepto fundamental para la teoría económica -por ejemplo: producción, intercambio, distribución, capital, trabajo- como hechos derivados. Con este enfoque se hace comprensible especialmente la diferencia entre valor y precio como un momento de la

\footnotetext{
${ }^{14}$ Cito los términos en inglés, debido a su mayor precisión. En la traducción alemana éstos corresponden a: Identität y Verschiedenheit. Ver Whitehead (1979: 69 y s.).

${ }^{15}$ La teoría de la estabilización podría ser más convincente a través de la idea del desequilibrio, según la cual se tendrían que producir o muy pocos bienes o demasiados, con lo cual el poseedor del dinero o el propietario de los bienes debieran decidir prácticamente solos si se produce un pago o no. Solo bajo esta condición es posible calcular sobre la base de relativa estabilidad. Ver al respecto Kornai (1971). Una sorprendente crítica al principio de equilibrio se encuentra también en el comienzo del debate de la ciencia económica (especialmente en relación a Montesquieu): El principio de equilibrio declara la inestabilidad como estabilidad, dos granos son suficientes para desestabilizar el balance. Así por ejemplo Linguet (1770: 96).
} 
diferenciación del sistema. Una vez que se producen pagos se necesitan precios que permitan formar expectativas sobre el importe a pagar y para comunicar al respecto ${ }^{16}$. La autopoiesis del sistema es por tanto independiente de un acuerdo acerca del "valor real" de bienes y servicios; y es sobre todo independiente de obligaciones de gratitud (y también del temor a la aparición de obligaciones de gratitud) que podrían resultar del hecho que uno de los lados diera más valor (o en su opinión diera más valor) que el otro.

Cada pago efectivamente realizado influye en la formación de precios y por lo tanto obtiene un efecto secundario en el nivel de formación de estructuras; junto con regenerar las posibilidades de pago en manos del destinatario, hace posible formar expectativas sobre qué pagos han de ser considerados para qué bienes y servicios ${ }^{17}$. Las regeneraciones de pagos por medio de pagos se forman como autoestructuras deliberadamente contingentes y variables, es decir son influenciables por interacciones (negociaciones) y organizaciones, ya que la autopoiesis de la economía está asegurada sobre la base del dinero.

También se puede entender en este marco teórico el criterio de la ganancia (y separarlo de la supuesta necesidad de gravamen a la plusvalía por parte de los "capitalistas"). La ganancia se produce cuando el pago beneficia al propio pagador. Los pagos hacen posible siempre, directamente y en primer lugar, los pagos de los demás. Solo el destinatario del dinero puede gastar de nuevo el dinero recibido. No obstante el sistema puede estar dispuesto de forma que indirectamente el pagador mismo gane también en opciones de pago. De este modo, la autopoiesis del sistema se convierte en un proceso reflexivo. Se dirige a sí mismo. Uno paga para poder actualizar y multiplicar sus propias posibilidades de pagar (en lugar de solamente obtener el objeto o el servicio por el cual se paga). Solo cuando el sistema acepta este criterio de ganancia como punto de vista para su autocontrol, este se hace independiente, en materia de producción, de motivos y estimaciones de valor "privados"18; se hace independiente, a saber, de la preferencia de alguien por la explotación de una fábrica de perfumes a una curtiduría, e independiente de la disposición o sentimiento de deber de alguien por continuar el negocio de su padre. Solamente el consumo se mantiene abierto a motivos privados (si es que no se orienta a las ganancias). Con anterioridad se había diagnosticado ciertamente al afán de ganancia como algo propio de la naturaleza -algo que proviene de Boccaccio- para hacer plausible el argumento de la falta de control de este impulso ${ }^{19}$. Una adopción facilitadora adicional a esto observó y legitimó las oportunidades de ganancias, especialmente en base al comercio exterior, y adoptó la forma de un efecto- modelo literario en el tema de los viajes de aventuras ${ }^{20}$. Con esto, la aprobación del nuevo motivo quedó desacoplada de los problemas de distribución internos al país y sus repercusiones políticas (y se puede ver al mismo tiempo que el umbral donde estaba la moral fue superado mediante la introducción de este principio recursivo de autolegitimación de la actividad económica). Durante un período transitorio (Luhmann 1980) podrían ser necesarias estas semánticas de avanzada [Lancier-Semantik], y en efecto el éxito en la búsqueda de ganancias produjo

\footnotetext{
${ }^{16}$ En detalle en Luhmann (1983).

${ }^{17}$ La doctrina del "precio justo" no fue otra cosa que la exigencia de atenerse a este fundamento de orientación. Ella no contenía ni una regla especial de cálculo para los precios (aparte de criterios puramente económicos, como la consideración de los precios de importación, los costos de los materiales y de la producción) ni tampoco una garantía de estabilidad de precios, sino que se dirigía solamente en contra del alza abusiva de los precios causada por dificultades de abastecimiento. Véase de Roover (1958).

${ }^{18}$ ¡No hay una errata acá! Por supuesto, estoy consciente que la teoría que quiere abolir al sector privado debe sostener lo contrario. Sin embargo, el sector privado ya ha sido abolido hace tiempo.

${ }^{19}$ Los estudios históricos que conozco no se remontan mucho más atrás. Los temas legales discutidos en el año 1600 apuntan a una antigua doctrina. Véase, por ejemplo Chalk (1951) y Ehrlich (1955).

${ }^{20}$ Véase Gunn (1969: 245 y ss.). Véase también Watt (1957: 63 y ss.). El contexto de la "aventura" hizo posible, en suma, una naturalización convincente del tema de las ganancias y al mismo tiempo una rutinización del héroe (Robinson Crusoe, Moll Flanders).
} 
riqueza. Pero con ello solamente se agudizaron los problemas de distribución que empiezan a preocupar desde el siglo XIX. El mismo afán de ganancias desprivatiza al que se dedica a él, y no obtiene su función de la extracción de "riquezas" de la economía, sino justamente en lo contrario: en la clausura autorreferencial del sistema funcional. Incluso las ganancias deben ser convertidas en pagos, y solo son ganancias cuando esto sucede.

Aparte del hecho que el sistema está clausurado por este motivo, independencias importantes en términos sociales, temporales y factuales se encuentran también asociadas. En la dimensión social, el sistema se independiza de la reciprocidad y por tanto de condiciones fuertemente influenciables causadas por el rango social de los participantes (Thurnwald 1936). Esta diferenciación normalmente esperable de la reciprocidad $^{21}$ hace a la economía autónoma, es decir, capaz de regularse por sí misma ${ }^{22}$. La ganancia es un motivo de aprobación independiente y no selecciona la acción a partir de la expectativa de que el otro se comporte de manera complementaria (como por ejemplo, que el vendedor de coches acuda al médico que ha comprado su coche con él). La ganancia es, con todos los condicionamientos sociales, menos vulnerable que la reciprocidad.

En la dimensión temporal y factual de la orientación hacia la ganancia, pueden ser aprehendidos objetos y procedimientos nuevos y no probados todavía. No se es dependiente de la legitimación del mundo ya existente y lo nuevo no es experimentado primordialmente como una desviación. En lugar de esto toma lugar un principio de selección más abstracto, el que puede actuar, tanto como una regla de detención para lo antiguo, así como una regla para la adopción de lo nuevo. El principio de clausura recursiva del sistema aumenta así sus grados de libertad mediante una elevada y simultánea selectividad.

\section{IV}

Ya sabemos cómo se reproduce la economía como sistema autorreferencialmente cerrado, a partir de los elementos que lo componen y con la ayuda de estos mismos elementos. Esto y no otra cosa constituye la unidad de la economía. Solo de este modo puede mantenerse en la sociedad como un sistema diferenciado y emplear recursividad para seguir reproduciéndose. No existen objetivos relacionados con esto, pues ello significaría que habría un final determinado, cuya obtención haría detener las operaciones de la economía ${ }^{23}$. La determinación de fines sigue siendo posible y relevante, incluso y especialmente en la economía. Pero los fines organizan siempre y solamente episodios en cuya finalización -una vez alcanzando el fin- se debe volver de nuevo a la capacidad de pago. La autopoiesis de la economía trasciende todos los fines económicos y los hace de este modo tener sentido (así como uno en la propia conciencia piensa frases o realiza cálculos, las reflexiones sobre los fines solamente pueden tener lugar porque uno puede estar seguro que al final de dicho episodio de reflexión y cálculo no cesará la autopoiesis de la conciencia). No se puede pensar hasta morir, y tampoco se puede evitar provocar con todos los pagos atados a fines también la reproducción de la economía en sí, porque

\footnotetext{
${ }^{21}$ Un paralelo en términos legales lo constituye la emergencia de los "derechos subjetivos". Ver Luhmann (1981, 1981a).

${ }^{22}$ Véase especialmente Polanyi (1978). Un malentendido -del cual Polanyi no es del todo inocente- debe ser por supuesto evitado. El problema de la diferenciación de la reciprocidad no tiene nada que ver con la cuestión de si y en qué medida la actividad económica se orienta por el interés propio del agente (aunque la semántica del interés propio acompaña a este proceso y lo co-posibilita). Centrarse en el interés propio es también perfectamente posible bajo condiciones de reciprocidad (como sabe cualquiera que regatea en el bazar), mientras que, por el contrario, el criterio de ganancia puede ser manipulado de manera puramente matemática y sin egoísmo.

${ }^{23}$ Ignoramos aquí conscientemente el concepto teórico trascendental de fin, el cual señala algo así como la interacción de las partes con el todo, la armonía interior o la unidad interna del sistema. Este concepto ha sido sustituido, al menos parcialmente, por el de autopoiesis, pues el primero fracasaría frente a las siguientes consideraciones teóricas relativas a la complejidad y la contingencia.
} 
de lo contrario el último pago sería totalmente imposible (¿quién lo recibiría?). La autopoiesis es un suceso autorreferencial y justamente por esto interminable.

Nuestra representación sin embargo está en cierto modo incompleta. Ella se ha ocupado de la clausura pero no de la apertura del sistema de la economía. El deseo de pagar por pagar es evidentemente un motivo defectuosamente organizado en el interior de la economía. No se paga (ni tampoco se ahorra) a menos que se tenga una razón. El sistema se obliga a sí mismo a encontrar razones, se obliga a sí mismo a través de su clausura para la apertura.

La apertura de la economía se refleja, por lo tanto, en el hecho que los pagos están vinculados a otros pagos por motivos que en última instancia se refieren al entorno del sistema. Esta es una idea intrincada que requiere un análisis muy preciso. Para ello emplearemos el concepto de necesidades.

Este concepto pretende describir una forma sistémico-económica interna para el procesamiento de la información. No es considerada por tanto como un "dato" del entorno, a pesar de que aparece de este modo en la economía. De lo contrario no se podría organizar en el sistema económico el lazo de la apertura con la clausura; la unidad operativa de los procesamientos de información y pagos. Se debe recalcar que esto no es menos importante, debido a que el orden de las necesidades que explicaremos más adelante no debe ser entendido como una jerarquía antropológica o psicológica entre motivos. Las necesidades se obtienen solamente de las diferentes relaciones con la economía y por lo tanto surgen también solo con, y a través de, la diferenciación del sistema económico. Evidentemente aquellas sociedades sin sistema económico diferenciado tienen que satisfacer también necesidades, pero estas no pueden surgir sin acción económica ${ }^{24}$ [ohne zu wirtschaften]. Solo la forma en la que se consideran las necesidades con respecto a motivos y posibilidades de satisfacción cambia y se hace más compleja en la medida en que un sistema económico se diferencia en la sociedad.

La semántica de la "necesidad" se debe entender también en relación con el sistema económico diferenciado. Ella se refiere al problema de la inclusión de toda la población en la economía. En las sociedades estratificadas las necesidades eran atribuidas solamente a los pobres y se limitaban de este modo a un problema especial de un mundo imperfecto. "Necesitar es ser pobre; y ser pobre es ser miserable" [Avoir besoin, c'est estre pauvre; et estre pauvre c'est estre miserable] se sostiene todavía en el siglo XVII (Desmarests 1661, vol. 1: 21). Solo cuando los estratos altos pueden preocuparse legítimamente de hacer dinero $^{25}$, es decir solamente cuando la diferenciación funcional se impone a la diferenciación estratificada, el concepto de necesidad puede adquirir la universalidad que señalaremos aquí y en lo sucesivo ${ }^{26}$.

En primer lugar se deben considerar las necesidades básicas de la reproducción humana, es decir, situaciones que constituyen también un entorno para la sociedad. Su percepción no supone una economía diferenciada. Las exigencias relativas a lo que hemos tomado aquí en consideración, pueden aumentarse considerablemente en base a simples requisitos mínimos de supervivencia. Existen también necesidades que surgen solamente si hay dinero disponible para permitir su satisfacción. Esto solamente es posible cuando la

\footnotetext{
${ }^{24}$ En otras palabras, la economía es y sigue siendo, diferenciada o no diferenciada, una función del sistema de la sociedad.

${ }^{25}$ Como tema de conversación para el salón en Francia en la segunda mitad del siglo XVIII, véase también sorprendentemente Senac de Meilhan (1787: 323 y s.).

${ }^{26}$ La transición comienza alrededor del 1690 en Inglaterra y avanza gradualmente tras la superficial diferencia entre ricos y pobres -en primer lugar con una evaluación positiva de las necesidades de lujo de los estratos altos y con la tesis antropológica de una ilimitada (lo cual solamente puede significar: orientada hacia el dinero) codicia de las personas, hasta la perspectiva de la orientación al consumo en la economía de mercado. Véase Appleby (1976).
} 
economía ya está suficientemente diferenciada como un sistema de pagos. Por último, también existen necesidades que están más estrechamente vinculadas a la economía en sí, especialmente las necesidades secundarias de la producción económica, es decir la demanda de energía, de materiales y mano de obra. Estas necesidades son descritas como necesidades propias de la economía, sin embargo esta categorización de necesidades indica también un entorno de referencia, el cual es considerado como un motivo para los pagos.

Las descripciones de las necesidades registran de este modo siempre un motivo para los pagos, debido a esto ellas son, en diversos grados, tanto artefactos de la sociedad como de la economía. De este modo, la economía se garantiza a sí misma su operación como sistema abierto y como sistema cerrado -y en función del tipo de necesidad, de manera más o menos dependiente de sí misma. Para las necesidades dependientes de capacidad de pago esto es en sí un viejo argumento ${ }^{27}$. Hoy en día las necesidades secundarias del sistema económico han adquirido un rol mucho más importante, como la demanda de energía, materiales y mano de obra. Cuando hablamos (con una simplificación todavía tolerable) de necesidades elementales, necesidades de lujo y necesidades de producción, en esta secuencia se encuentra al mismo tiempo una creciente dependencia de la economía de sí misma. O formulado de manera más precisa: la dependencia del entorno por parte del sistema está condicionada por la medida en que el propio sistema se adapte, desde la satisfacción de necesidades elementales hacia la satisfacción de necesidades de lujo y, a continuación, a la satisfacción de necesidades de producción. En todos estos casos, la reproducción autorreferencial de los pagos a través de pagos une en su base a la clausura y a la apertura, en tanto exista una economía monetaria. Pero la combinación forzada de estos dos aspectos cambia su carácter en la medida en que la apertura es a su vez dependiente de la economía y con ello la reproducción de la capacidad de pago se hace dependiente de la reproducción de capacidad de pago. Luego en esta situación, cuando la economía no se las puede arreglar consigo misma, apela a la política. Entonces el intento de desautologizar la autorreferencia mediante autorreferencia está condenado a fracasar. La economía se adhiere a una instancia externa la cual no suspende con su acción la autopoiesis del sistema económico, sino que la garantiza.

Este último paso hacia la dependencia de la dependencia del entorno del sistema a partir del sistema se realiza con la transición a la sociedad industrial y desde entonces se hace prácticamente irreversible. Esto significa, entre otras cosas, que incluso el suelo (así como el resto de los recursos) y el trabajo solo se pueden basar en dinero. Solamente ahora la economía es un sistema integrado monetariamente y, como tal, se diferencia en todo lo que respecta a su propia reproducción. La sociedad renuncia a hacerse responsable de su propia economía y ya no hay tampoco instancias, es decir estratos altos, donde se pueda exigir dicha responsabilidad. El estereotipo negativo de los "capitalistas" se corresponde exactamente con esta situación. El capitalista es rechazado, pues no se percibe para él ninguna función en los estratos altos, él no representa a la sociedad, sino que solamente se ocupa de concentrar la capacidad de pago. Sin embargo la política, según todo lo que sabemos hoy en día, tampoco podría siquiera hacer algo más que esto.

\section{V}

En una teoría de sistemas establecida tan ampliamente no se puede contestar la pregunta relativa a la función de la economía en referencia a la satisfacción de necesidades (aunque solo se trate de necesidades "materiales"). Resulta tan evidente que las necesidades juegan un rol en la economía y que además estructuran la apertura del sistema y sus servicios hacia el entorno, es decir, están tan condicionadas por la propia economía, que

\footnotetext{
${ }^{27}$ No fue el primero, pero sí es representado de manera particularmente impresionante por Mandeville (1924). Más evidencia se encuentra en la introducción de Kaye y Appleby (1976).
} 
se podría ver en su satisfacción la función del sistema económico. En una sociedad funcionalmente diferenciada se debe hacer una distinción fundamental entre las relaciones entre los subsistemas individuales y las relaciones de dichos subsistemas con la sociedad. En el primer caso se trata de prestaciones en las cuales los subsistemas se orientan de manera adaptativa y enfocada en el aprendizaje, y de acuerdo a lo que el entorno intrasocial requiere de ellos. En la relación con la sociedad, en cambio, éstos son autónomos ya que son, por así decir, jueces de su propia causa, es decir salvaguardan una función para la sociedad ${ }^{28}$. La satisfacción de las necesidades puede por tanto considerarse, en el mejor de los casos, como una prestación de la economía. Con ello no ha sido aún respondida la pregunta por la función de la economía.

Recurrimos para esto, por ahora, a un argumento similar a aquel de la teoría política de Thomas Hobbes. Sociedad significa que las personas, en la determinación y satisfacción de aquello que experimentan como necesidad, no operan ni solitariamente ni de manera independiente entre sí. Cada persona estimula a la otra y ambas se interfieren mutuamente. Desde aquí se produce -y no desde la inestabilidad de la naturaleza- la necesidad de adquirir provisiones. Todos deben -porque otros ya están interesados y van a interferir- tomar provisiones a largo plazo y esto hace escasos a todos los bienes, pues todos quieren reservar para su futuro lo que otros necesitan en el presente ${ }^{29}$. Con el aumento incesante temporalmente de bienes almacenables aumenta también la escasez y debe inventarse un mecanismo social que enlace a una provisión estable en el futuro con cada distribución actual. Esta es la función de la economía.

Visto en términos formales, todas las economías se orientan por la escasez. La relación con la escasez no basta sin embargo como indicación de la función. De esto se deduce que una economía totalmente monetarizada tiene que arreglárselas, no solamente con una, sino con dos escaseces: la escasez de bienes y servicios mundialmente determinada y la escasez artificial de dinero. A esto lo hemos llamado anteriormente "codificación". La función de la economía por tanto debe ser cumplida mediante el condicionamiento de las relaciones entre ambas escaseces, especialmente mediante precios. Su función no puede entenderse simplemente como la reducción de la escasez o el aumento de la riqueza. La escasez no es en ningún caso una mera "fórmula de contingencia" que, interpretada como suma constante y como regla, cuesta algo a todo consumo y aliviana el traspaso del problema de referencia a las operaciones y las regulaciones (Luhmann 1972). Esto podría justificar la definición del objeto de la ciencia económica (y en especial: el objeto de autorreflexión del sistema económico) como una disposición de bienes y servicios escasos $^{30}$. No obstante, las fórmulas de contingencia son siempre reducciones que toman el lugar de la función cuando se trata de la orientación del sistema hacia sí mismo. El análisis socio-teórico debe por tanto al menos dirigirse hacia la función real, y esta consiste precisamente en la producción y regulación de la escasez para la desproblematización de una futura satisfacción de necesidades. El problema de referencia de la economía es, con otras palabras, el futuro- presente; se podría decir también, la irritabilidad del presente por el futuro, o el problema social del padecimiento actual de escasez causado por otros ${ }^{31}$. El problema surge del hecho que la dimensión temporal y la dimensión social son transversales entre sí y se afectan mutuamente.

\footnotetext{
${ }^{28}$ Respecto de la aplicación de la misma clasificación para el caso de otros sistemas funcionales, véase Luhmann (1981b; 1977: 54 y ss.) Luhmann \& Schorr (1979: 34 y ss.) y Luhmann (1981c: 81 y ss.).

${ }^{29}$ Véase también al respecto Belshaw (1965: 110 y ss.): la reintroducción de la escasez en la diferenciación interna del sistema económico.

${ }^{30}$ Una idea ampliamente compartida. Véase solamente Rees (1968: 472) y Montias (1976: 81 y ss., 83).

${ }^{31}$ Esto tiene ciertamente dos lados: (1) los otros se apropian de algo que uno mismo quisiera tener, y (2), los demás no trabajan lo suficiente. El problema, si bien se formula de manera neutra respecto de los estratos, tiende a hacer una diferencia entre ellos.
} 
La función de la economía así definida permite clarificar las ventajas que ofrece la diferenciación de un sistema económico. La función especial de la economía es proporcionada mediante su propio sistema autopoiético. En este sistema los pagos posibilitan pagos. Como resultado se construye un futuro, en principio, ilimitado. Todas las disposiciones del sistema aseguran al mismo tiempo el futuro del sistema. Más allá de todos los objetivos, todas las ganancias, toda satisfacción, el sistema continúa. El sistema no puede darse término a sí mismo, pues el sentido del dinero reside en gastarlo. La garantía que confiere la facultad de gastar (bajo condición que la aceptación de dinero aparezca como recompensa) no puede otorgar por sí sola una seguridad abstracta para el futuro -bajo la "forma de mercancía"-, y la estimación relativa a la propiedad, capital, lugar de trabajo y derechos a jubilarse se subordinan a este objetivo. Desde todas estas perspectivas se trata de participar en la autocontinuidad de la economía y el criterio de ganancia sirve solamente, como hemos mostrado, para volver a relacionar esta recursividad consigo misma.

Con esto hemos solucionado sin embargo solamente la mitad del problema. No se trata únicamente de la satisfacción permanente de necesidades, sino también de prioridades temporales entre diferentes necesidades, de diferentes personas o sistemas sociales. Se trata también siempre de problemas de distribución actuales que demandan decisión (en realidad: ison decisivos!), y la seguridad futura no reside por último en perspectivas favorables de participar ahora y en el futuro de la distribución. El concepto de clase está disponible, desde Quesney, para describir la distribución de personas en la distribución de seguridad futura. Saint- Simon y Marx continuaron esta tradición (Luhmann 1983a [1985]). Pero el concepto de clase solamente considera la reflexividad de la distribución, la distribución de distribuciones, y no el propio proceso basal y por esto nunca logró convencer por completo a los economistas.

En la teoría aquí presentada el problema de referencia de la función de la economía puede ser identificado con la unidad entre clausura y apertura del sistema económico. La función es, con otras palabras, la especificación histórica y social de la unidad entre clausura y apertura del sistema, y solo porque esto sucede de este modo la sociedad se estructura según el principio de la diferenciación funcional. Esto se aplica mutatis mutandis para otros sistemas (Luhmann 1983b). Para el caso de la economía, la unidad abiertaclausurada de reproducción autopoiética se especifica de modo que la clausura del sistema (bajo las condiciones del valor del dinero, esto es, bajo condiciones de aptitud de permitir pagos para otros pagos) garantiza la seguridad en el futuro bajo la forma de capacidad de pago. Allí reside también la garantía que aquél que puede pagar puede satisfacer sus necesidades. La apertura del sistema garantiza, al mismo tiempo, que todos los pagos se orienten a satisfacer necesidades y que cualquier persona que quiera satisfacer sus necesidades dentro del rango aumentado por el dinero, deba pagar, es decir, deba hacer posibles los pagos. Visto empíricamente, creo que no caben dudas que esto funciona de este modo. El problema se ubica en las condiciones iniciales, históricas y sociales, y en sus efectos secundarios.

El nivel de rendimiento de la economía diferenciada y su autopoiesis monetariamente controlada constituye un logro altamente improbable y por tanto inestable. La separación resultante de la política es, por ejemplo, políticamente difícil de sobrellevar. La investigación científica ve también amenazada su autonomía, cuando por un lado ella demanda cada vez más dinero y, por otro lado, solamente produce un porcentaje muy reducido de resultados que pueden ser utilizados provechosamente bajo el principio selectivo de la economía. Dichas tensiones se ven agravadas debido a que aunque la economía lleve a cabo sus funciones, aparentemente tiende sin embargo a realizar una desigual distribución, es decir entrega más a los "ricos" (sobre todo más crédito y más 
oportunidades para explotar la movilidad de sus inversiones) que a los "pobres"32. Por último, el valor del dinero es un medio de resolución de problemas altamente sensible y resulta evidente que puede ser puesto en peligro fácilmente por las inflaciones y deflaciones. Debemos dejar hasta acá estas consideraciones. Ellas sirven por el momento solamente para rechazar la idea que el trasvasije del cumplimiento de funciones en sistemas autopoiéticos de la sociedad debiera ser celebrado abiertamente como un progreso. Hoy en día difícilmente sabemos si sobre esta base evolucionará una estructura social permanente (o al menos estable durante algunos siglos). Los movimientos socialistas tienden, especialmente donde logran imponerse políticamente, a hacer frente a estos problemas y los reducen a una diferencia entre presente y futuro transversal a toda la sociedad. Esto apenas puede ser considerado una descripción adecuada de la sociedad. Nos devuelve más bien al principio. Se hace entonces aún más importante describir mediante los conceptos adecuados lo que ha sucedido y lo que está sucediendo.

\section{VI}

Se puede dar un paso adicional para una mayor clarificación, si se toma en cuenta la sugerencia de Talcott Parsons y se concibe al dinero como un medio simbólicamente generalizado que, de manera similar a las operaciones del lenguaje, es controlado por un determinado código. No nos adheriremos estrictamente a la arquitectura teórica parsoniana, sino que no situaremos de acuerdo con la tesis que señala que los sistemas sociales, incluida la sociedad, constan de comunicaciones, y en lugar de referirnos a "medios de intercambio" hablaremos de "medios de comunicación simbólicamente generalizados" ${ }^{\prime 3}$. En este cambio de perspectiva teórica se produce una inversión: los medios de comunicación no son el resultado de la diferenciación funcional sistémica, sino más bien son catalizadores para la diferenciación de sistemas funcionales ${ }^{34}$.

El punto de vista funcional para este segmento teórico surge del hecho que toda comunicación produce, en primer lugar, una situación abierta en la cual son posibles tanto la aceptación como el rechazo de una propuesta de sentido. Los medios refuerzan la probabilidad de aceptación para situaciones donde se espera más bien un rechazo. Ellos aumentan la posibilidad que la comunicación tenga lugar. Impiden que la probabilidad de rechazo del proceso de comunicación desaliente y motivan, en cambio, por la manera en que la selección se presenta para la comunicación.

Esto puede ser compatible, cómo hemos mostrado antes para el caso del dinero, con una gran libertad para aceptar y rechazar. El código del dinero no obliga a nadie a vender o a prestar servicios, sino que permite combinar la demanda con una oferta de pago y esto, a su vez, hace posible ajustar ofertas de objetos y servicios e incluso organizaciones completas para que tal demanda se produzca. Dicho de otra manera, los productores se benefician de que la natural improbabilidad de la demanda por sus bienes se haya disipado. El efecto más importante del medio dinero surge en el nivel transversal de la sociedad y se debe al hecho que el pago tranquiliza a los demás. Usted puede tolerar ver a alguien acceder a bienes escasos, a pesar de que usted mismo esté interesado en tales o cuales productos y servicios (o en el futuro pueda estar interesado), debido a que él paga. Y usted puede también aceptar que esto suceda bajo condiciones en las que las negociaciones entre las partes del contrato ocurran sin su participación, debido a que la contraprestación en dinero ocurre de una manera tal que regenera al medio, debido a que este solo es utilizable bajo la forma de transferencia. La selección de una acción, a saber,

\footnotetext{
${ }^{32}$ Que esto también afecta las condiciones de las economías nacionales (con la inclusión de los problemas coloniales), lo sabía ya Hegel. Solo basta ver las impresionante notas finales a sus lecciones dedicadas especialmente a este respecto (Hegel 1983).

${ }^{33}$ Véase en general: Luhmann (1975a, 1981d).

${ }^{34}$ Esto también tiene consecuencias evolutivas, cuyo análisis por el momento debemos posponer. Algunas alusiones sobre esto se encuentran en Luhmann (1975b).
} 
el acceso a bienes escasos, es transformada por el código del medio de dinero en una mera vivencia de los demás. Se acepta como información acerca de un hecho en el que no se está involucrado.

El medio dinero asegura, por tanto, que en el ámbito de la economía -a pesar de la divergencia latente de intereses- la acción tenga para el observador aproximadamente el mismo sentido que el que tiene para los propios actores. Las divergencias no son llevadas al extremo y la ruptura de las antiguas condiciones locales no conduce inmediatamente al conflicto. El interés propio de los terceros es neutralizado o desviado a su propia participación en la autopoiesis de la economía. Los demás se ven motivados a hacer sus propios negocios, a pagar por sí mismos para obtener y poder pagar, y de este modo se compensa de una forma altamente generalizada que cualquiera esté interesado en el pan y el vino, en la jardinería y en la reparación de sus zapatos, y que alguien no comprenda por qué deba dar a otros prioridad en tales cuestiones.

La diferenciación de esta prestación relativa a la transferencia de selectividad desde la acción hacia la vivencia logra tener éxito si la condición de escasez (que hace improbable la transferencia) es simulada en el código del medio. El dinero en sí se debe considerar como escaso, y aun cuando se sabe que este como medio de comunicación en sí no puede ser escaso. Al mismo tiempo, se obtiene la ventaja adicional relativa a que la escasez se puede cuantificar y se la puede operacionalizar como principio de suma constante. Todas las réplicas a este principio, desde los intereses monetarios hasta las manipulaciones de sumas de dinero, se subordinan a esto: si se gasta una moneda se tiene una moneda menos -ni más ni menos. Y si se recibe una moneda, se tiene una moneda más, nada menos y nada más**. Este principio controla el proceso de pago, a saber mediante noidentidad del cálculo en ambos lados (especialmente las necesidades y motivos).

La escasez es de este modo, por una parte, una fórmula de contingencia incorporada en el código del medio, la que considera que todo es posible de otro modo y otorga una forma tecnificada disponible para cada operación. La escasez es también una segunda versión de la función del sistema económico, aquella relativa a distribuir seguridad en el futuro. De manera exactamente igual, el pago en dinero realiza dos niveles de sentido en uno: se orienta al medio simbólicamente generalizado del dinero y también reproduce al sistema autopoiético de la economía. Este hallazgo sugiere una convergencia entre la teoría de los medios de comunicación y la teoría de sistemas. Los medios de comunicación ayudan a atravesar un umbral de improbabilidad que surge de aquellas condiciones mencionadas de enlace comunicativo entre selecciones ${ }^{35}$.

Esta función se puede combinar con la formación de un sistema autopoiético que utiliza el medio como un código para su autorreproducción. Esto no constituye una adquisición inevitable, ni a nivel lógico ni evolutivo (tal tesis estaría, por lo menos, más allá de lo que podríamos fundamentar). Pero si esto tiene éxito, se produce un sistema dotado de un alcance especial en la sociedad. Todas las comunicaciones que utilizan el medio pueden diferenciarse y reproducirse en un sistema cerrado, donde se asumen las condiciones de reproducción en el propio código del medio. La comunicación se emplea para darse una oportunidad de éxito, como una estructura que permite al mismo tiempo la formación y reproducción recursiva de los elementos que diferencian al sistema. En el caso del dinero se puede mostrar cómo esto es posible.

\footnotetext{
** [N. del T.] El texto original habla de "marcos", la antigua moneda alemana actualmente en desuso.

${ }^{35}$ La constelación regulada por la propiedad y el dinero, en la cual las acciones de unos deben ser aceptadas como vivencias de otros, por supuesto, constituye solamente uno de varios problemas posibles. Para otros casos, como la verdad, el amor y el poder, véase Luhmann (en Habermas \& Luhmann 1971: 352 y ss.), también Luhmann $(1975,1982)$.
} 
VII

El hecho que la economía produce su diferenciación en la sociedad con la ayuda del dinero y en dirección hacia una autorreproducción autopoiética de pagos a través de pagos, hace finalmente comprensible la tipicidad de la diferenciación interna del sistema económico. Nos topamos aquí con un tema que ha sido tratado clásicamente desde un punto de vista de las ventajas de la división del trabajo ${ }^{36}$. Sin tratar de refutar directamente esta postura, reemplazaremos, no obstante, la perspectiva del aumento de eficiencia por medio de una pregunta distinta.

Autopoiesis significa para el sistema: producción continua e interminable de elementos del sistema a través de elementos del sistema. Todos los complejos operacionales terminables constituyen solamente episodios y desembocan nuevamente en la apertura hacia nuevos fines que sirven a la autocontinuación del sistema. La unidad del sistema no puede tener un objetivo, ya que se entiende a sí misma como autorreproducción, y la condición para esto es que nada está sucediendo. Cuando la autopoiesis en cambio está asegurada (o se puede suponer que lo está), se hace posible una mayor arbitrariedad en la definición de fines y en los arreglos entre medios/fines -solamente porque el "fin" no representa la "unidad interna" del sistema, sino solo un episodio entre muchos que llega a su término. El relacionamiento recíproco entre fines y medios puede entonces ampliar y reestructurar las contingencias internas puesto que la unidad del sistema está asegurada y no tiene dependencias ${ }^{37}$. O dicho de manera más fina: debido a que el sistema está clausurado de manera recursiva, puede estructurarse internamente en episodios y en relación con ellos permitir contingencias altas y capaces de descomponerse nuevamente ${ }^{38}$. Los fines pueden también desacoplarse de la idea de una finalización natural de procesos naturales y pueden ser pensados como seleccionables ${ }^{39}$.

En el sistema de la economía monetaria, la diferenciación interna se puede introducir, por tanto, a través de una repetición, agregación y diversificación de los fines -solo bajo la condición de que la persecución de fines produzca algún rendimiento. Esto ocurre solamente en el caso de la producción, no así en el consumo. En el primero, el aumento de la complejidad se encuentra en el ámbito de industrias o actividades directamente relacionadas con la producción y sus empresas asociadas, especialmente el comercio y otros servicios. Solo aquí se pueden formar, en estricto rigor, subsistemas. Mientras que el consumo, por su parte, a pesar de ser una actividad económica -pues cuesta dinero- se encuentra disperso en toda la sociedad. Ciertamente la división funcional entre producción/distribución/consumo domina la orientación del sistema económico. Las diferencias respectivas son las diferencias directrices del sistema (y no por ejemplo la diferencia entre ricos y pobres). En ellas encuentra su apoyo la necesidad de dinero. Sin embargo estas no pueden desarrollarse en dirección a una diferenciación de

\footnotetext{
${ }^{36}$ Uno de los principales puntos de discusión es, y sigue siendo, este punto de partida: si se pueden considerar las ventajas de la división del trabajo como su función o incluso como causa de su desarrollo histórico, o si fue solo más tarde que se descubrieron los efectos secundarios que estabilizaron un patrón configurado previamente. En cualquier caso, este enfoque teórico produce una especie de idea de progreso del desarrollo histórico de manera casi inevitable incluyendo a Marx y a Durkheim.

37 En la teoría general de los sistemas autopoiéticos, esto se expresa en la terminología que distingue entre “organización” (clausura) y “estructura". Ver Maturana (1982: 277 y s.; 282 y s.) (referido allí al sistema nervioso).

${ }^{38}$ El argumento es exactamente el mismo que hemos utilizado anteriormente en la sección II: debido a que está cerrado, está abierto. En un caso se trata de diferenciación externa, en el otro de diferenciación interna.

${ }^{39}$ Aquí también se hizo necesaria por lo demás una semántica de transición, la cual tuvo que calmar la preocupación respecto que cada orden pudiera disolverse en el desarrollo contingente de los fines. La "mano invisible" y la "astucia de la razón" se volvieron particularmente conocidas. Sin embargo también los esfuerzos por subsumir lo general en lo particular, y en especial la crítica del juicio de Kant pueden ser leídos en este contexto. No es casualidad que el concepto y la teoría de la organización de los siglos XVIII y XIX hayan visto acá uno de sus puntos de partida y, consecuentemente, hayan distinguido terminológicamente en primer lugar entre orden y organización.
} 
subsistemas ${ }^{40}$. En este sentido, en la realidad de los sistemas sociales no existe tampoco un equivalente para los conceptos de equilibrio de la teoría económica (lo que por supuesto no es una objeción para su uso analítico).

Esto tiene consecuencias para el concepto de mercado. Se podría pensar preliminarmente que el mercado es un sistema en sí -como por ejemplo: que lo que sucede en el mercado de la plaza junto al ayuntamiento podría continuar, con el debido progreso racional, finalmente en el supermercado. El mercado sería entonces el conjunto de organizaciones de distribución o roles individuales que median entre la producción y el consumo. Pero si se mira más de cerca, se observa que también estas organizaciones "se orientan al mercado", y esto significa evidentemente que no se orientan a sí mismas. El "mercado", por lo tanto, no es otra cosa que un límite, es la percepción del consumo desde el punto de vista de la producción y las organizaciones de distribución ${ }^{41}$. Sumado a esto, el mercado aparece también como los esfuerzos de los competidores, siempre y cuando éstos influyan en las oportunidades de venta. Visto así, la producción se presenta a sí misma como mercado. El límite actúa como un espejo que contribuye de este modo a la integración de la producción, como cualquier empresa que en el espejo del mercado obtiene su propio reflejo y el de sus competidores (y de sí misma, como un competidor de los demás competidores). En este espejo los consumidores aparecen como escasos. Según esto, hay muy pocas necesidades y justamente por eso se recomienda la sobreproducción (es decir, idesequilibrio!), de modo que uno se prepare ante cualquier eventualidad que permita aprovechar las oportunidades de ventas disponibles.

Formulado de manera abstracta, el mercado como límite es la diferencia entre complejidad definida e indefinida (propia y relativa al entorno). La propia complejidad es y como sociólogo uno solo puede decirlo de manera vacilante- controlable a través de la organización. Posee una seguridad histórica como inversión y una consiguiente capacidad de cambio limitada. La complejidad del entorno es por el contrario indefinida -debido a, por un lado, la variedad de sus posibilidades interdependientes y, por otro lado, también y especialmente porque no se puede determinar de manera independiente de las actividades de los competidores y de las propias actividades como un competidor de los demás competidores. Por lo tanto, el entorno aparece para el mercado en la forma de un círculo autorreferencial, o bajo la forma de doble contingencia: lo que uno hace, depende entre otras cosas, de lo que uno hace ${ }^{42}$. Esto es lo que parece premiar al empresario innovador de Schumpeter, cuando un número suficiente de otros empresarios innovadores fracasan.

Como cualquier efecto de espejo este se ve afectado por opacidades, a saber, por la opacidad de la complejidad indefinida. Se pueden ver y evaluar las conexiones para las acciones propias y adaptarse al medio ambiente mediante la transformación de la incertidumbre en riesgo. Esto solo podría ser en principio modificado si el Estado establece otro espejo en el que los consumidores se visualicen a sí mismos en competencia por mercancías y estas se perciban a su vez como escasas. En lugar de un sobreabastecimiento se simula entonces un abastecimiento insuficiente, junto con una combinación diferente de trastornos. En última instancia el problema parece originarse en la forma de diferenciación interna de la economía. No se realiza a la manera de una descomposición de una unidad determinada en diversos sub- sistemas (como cuando se dice que la ciencia se diferencia en disciplinas). Más bien, surge a través de la diferenciación de un subsistema altamente complejo para la producción, cuya propia complejidad se proyecta en un entorno correspondientemente indeterminado. Si esto no

\footnotetext{
${ }^{40}$ Debo corregir los supuestos correspondientes que aparecen en Luhmann (1970: 219 y ss.). Resulta particularmente engañoso considerar al consumo económico primariamente como tema de unidad doméstica y de este modo ocultar la producción del propio consumo. Las consecuencias para el concepto de mercado deben ser reconsideradas.

${ }^{41}$ Sobre esto y sus consecuencias resulta muy interesante White (1981).

${ }^{42}$ Es decir, "entorno turbulento" en el sentido de Emeryt \& Trist (1965).
} 
cambia, el mercado conserva su función como articulación de la diferencia entre complejidad definida e indefinida.

\section{VIII}

La unidad de un sistema autopoiético no es otra cosa que la producción de sus elementos a través de sus elementos. La unidad del sistema económico es el hacer posible pagos a través de pagos. Este es el punto de partida. Este es también el punto de partida de todo conocimiento, de lo contrario perdería de vista su objeto. De este modo, se responde al problema (como tal criticable) de la constitución de la unidad del objeto, pero con esta respuesta generamos al mismo tiempo un conjunto de problemas adicionales y necesidades de distinción, las que se relacionan con las condiciones de posibilidad de las "ciencias económicas" y su emplazamiento en la sociedad.

En primer lugar, en cuanto las ciencias económicas demandan ser ciencias, ellas mismas se hacen parte de un sistema autopoiético socialmente diferenciado. Su operación basal es la obtención de conocimiento ${ }^{43}$. Este sistema produce conocimiento a partir de conocimiento y lo califica como conocimiento, en tanto dicha calidad pueda ser aceptada en la relación recursiva con otros conocimientos. Esta producción se lleva a cabo a su vez en un sistema cerrado, solo cuando el estado de desarrollo de la sociedad permite que únicamente el conocimiento pueda intervenir en la calificación de los elementos como conocimientos. Con esto el entorno (en nuestro caso, la economía) aparece como un objeto de conocimiento. Este sistema está también cerrado y abierto al mismo tiempo, es decir, abierto en base a su clausura. De manera funcionalmente similar a lo que hemos mencionado antes acerca de las "necesidades", la categoría de "objeto" sirve aquí para presentar la apertura al entorno en un sistema recursivamente cerrado. Del mismo modo como sucede con las necesidades, si esta forma de orientación ha de cumplir su función de abrir un sistema cerrado hacia su entorno, los objetos no pueden ser arbitrariamente elegidos o distinguidos. Si se trata de un sistema autopoiético como objeto (lo que para la ciencia constituye evidentemente solo una entre muchas posibilidades de concebir sus objetos) se requiere que la terminología (es decir, ino se trata de la realidad misma!) considere que este tipo de objetos produce por sí mismo su propia unidad (vale decir, no se trata de un mero artefacto analítico o clasificatorio). Por lo tanto, se torna relevante para la ciencia económica prestar atención a la manera como el objeto economía produce su propia unidad.

A partir de esto se debe distinguir lo que bajo la forma de autobservación y autodescripción se desarrolla en el sistema económico. Se trata aquí de cómo se comunica en la economía acerca de la economía ${ }^{44}$. A partir de las operaciones elementales de pago se distinguen autobservación y autodescripción, dado que se relacionan con las estructuras a las que se orientan los pagos, es decir, los precios. Se orientan, al igual que toda autobservación y autodescripción, hacia diferencias, las cuales aquí se traducen en diferencias de precios. Normalmente se trata de comparaciones de precios, incluyendo comparaciones diacrónicas, es decir se trata también de aumentos o disminuciones. A esto se puede relacionar -nuevamente de manera agregativa- un lenguaje; las diferencias entre precios, esto es, aumentos o caídas de los precios, no se reportan como meros hechos, sino que se busca calcularlos, explicarlos o modificarlos. Los más antiguos intentos de articular este nivel sirvieron parcialmente para un control de los compradores. Los principales ejemplos de esto aparecieron en el contexto de la discusión elaborada en

\footnotetext{
${ }^{43}$ Deliberadamente decimos no solamente conocimiento, sino obtención de conocimiento. Véase al respecto Luhmann (1981e).

${ }^{44}$ En relación con sistemas sociales, "observación" y "descripción” corresponden siempre a comunicación y no por ejemplo a actividades psíquicas per se. Los sistemas sociales no disponen de otros tipos de operaciones elementales y deben asimismo producir en el sistema, y con los medios del sistema, las operaciones de autobservación y autodescripción.
} 
torno al "precio justo" en la escolástica y la escolástica tardía, así como paralelamente en las reflexiones acerca de todo lo que se debe observar para una regulación política de los precios. Sería históricamente erróneo ver esto como una especie de "prehistoria" de la ciencia económica ${ }^{45}$; más bien caracteriza lo contrario: se trata de un debate moral- legal que está dominado por la diferencia entre derecho y no-derecho, el cual presenta las relaciones económicas a lo sumo desde el punto de vista del éxito o fracaso de determinadas medidas (Grice- Hutchinson 1978).

Esta reflexión varía solamente en el siglo XVIII (hasta entonces valían los medios de argumentación escolástica). Resulta evidente que este cambio estuvo relacionado con la diferenciación, llevada al extremo, de un sistema económico reproducido en base a dinero. Solo entonces se da comienzo a una ciencia económica que observa ante sí a su objeto como un sistema autopoiético -aunque no con este concepto. Solo entonces surge también en la propia economía una necesidad de reflexión, junto con el esfuerzo de entender operativamente las estructuras y los cambios estructurales del sistema como unidad del propio sistema. Pronto uno debe abstenerse de especificar esta unidad como una unidad doméstica o como sociedad civil bajo la forma de un Estado. En vez de esto se debe entender el objeto en base a tipos de operación, tales como el intercambio, la producción o la distribución, para los cuales no existen correspondencias en el entorno del sistema. Conforme a esto, la semántica de la naturaleza se convierte en semántica de libertad (a pesar de que el fin de la naturaleza y de la libertad permitió dar el salto).

Cuando en la economía se habla de la economía, sigue empleándose evidentemente un lenguaje completamente ateórico. Al lenguaje relativo a "precios" se le añade un lenguaje relacionado con el "capital". Se puede hablar de ingresos [Umsatz] y del crecimiento de estos, de la relación entre el capital propio y los ingresos, o del capital propio y el endeudamiento, y se pueden identificar con ello éxitos y fracasos; riesgos o garantías suficientes. Las diferencias por las que uno se orienta pueden residir en comparaciones temporales, o también en relación con pagos que son "propios de la industria". Este tipo de orientación debe considerarse en relación con el alto grado de descentralización de la toma de decisiones económicas y ello parece ser suficiente; pues para una comunicación orientada hacia la teoría faltaría, de todos modos, muy prontamente la información necesaria.

Sin embargo, visto en términos generales, junto con la elaboración de autodescripciones teóricas se añade un nuevo momento. Por lo tanto, debemos distinguir entre la autobservación (vivencia actual) y la autodescripción (elaboración de artefactos semánticos). Las teorías afectan al sistema que describen. Influyen en la política económica, en el comportamiento de inversión, etc., y esto sucede muy rápidamente ${ }^{46}$.

El ejemplo más evidente, cuya claridad no puede ser superada por otro ejemplo, se encuentra en los inicios: en la teoría económica de los fisiócratas ${ }^{47}$. Se reconoce la autonomía de la economía. Dicha autonomía se atribuye al intercambio mediado por el dinero, el cual incluye todo lo que es económicamente relevante y produce la interdependencia completa de todos los sucesos económicos. Esto fue para aquél tiempo una idea novedosa (la que sin embargo fue representada fuera de la fisiocracia) ${ }^{48}$. Ella fue descrita por medio de la metáfora del círculo y la circulación ${ }^{49}$ (algo totalmente irrealista,

\footnotetext{
${ }^{45}$ Al respecto, acertadamente Krauth (1982).

${ }^{46}$ La observación y la crítica de este fenómeno comienza también casi simultáneamente con su primera aparición. Véase el tratamiento de los fisiócratas como "secta" por parte de Linguet (1770: 14 y ss.) y adicionalmente por Mably (1768).

${ }^{47}$ Como una presentación muy cercana al punto de vista elegido aquí, véase Meek (1963: 364 y ss.).

${ }^{48}$ Véase, en términos programáticos, a Morellet (1980).

${ }^{49}$ Igualmente irrealista como el caso de aparato circulatorio sanguíneo que probablemente habría servido de modelo. Quesnay era médico. En realidad, por supuesto, ni el flujo sanguíneo ni el flujo de dinero adquieren la forma espacial de un círculo.
} 
desde un punto de vista descriptivo). Este sistema de circulación se supone a sí mismo; se mantiene, aumenta o reduce a sí mismo mediante la extensión o contracción del diámetro del círculo $^{50}$. El punto de partida es por tanto una metáfora: una imagen de simetría autorreferencial. En dicho modelo de economía han de ser incluidas, sin embargo, las asimetrías, el círculo debe romperse por interrupciones de sus interdependencias y en relación con el entorno del sistema ${ }^{51}$. Esto ocurre como si se tratara de tomar en cuenta las necesidades con un mínimo de concesiones teóricas y solamente en un lugar, específicamente allí donde la naturaleza interviene en la producción y a través de dicha intervención hace posible que existan excedentes, vale decir: en lo relativo a la tierra como factor de producción. Todos los demás factores (incluyendo el capital y el trabajo) son tratados como inmanentes para la economía. Ellos solo pueden reproducirse a sí mismos, porque se encuentran en el aparato circulatorio de la economía. Los excedentes solamente pueden producirse por una interacción entre el sistema y el entorno.

Esta teoría se ubica a sí misma como un conocimiento de su objeto economía y sostiene empecinadamente su propia "evidencia". Sin embargo arrastra al mismo tiempo consecuencias prácticas. Ella demanda eliminar los obstáculos políticos y legislativos de la economía que yacen en el sistema de impuestos y en las restricciones relativas al comercio y a los gremios ${ }^{52}$. Es posible suponer que esto que se ofrece como una consecuencia de la teoría es en realidad secretamente su propio planteamiento del problema -al igual que la cuestión del desempleo para Keynes. La teoría tiene objetivos político- económicos. Busca el aumento de la riqueza de la que todos participarán como propietarios y el monarca lo hará como propietario de la soberanía. Ve el orden legal existente ahora con nuevos ojos, como una restricción nociva que debe evitarse. La economía diferenciada a través del mecanismo del dinero posibilita, por un lado, nuevas percepciones, nuevas reflexiones de su unidad, nuevas semánticas y, por otro lado, las teorías correspondientes se reintroducen en el sistema como conocimiento y buscan intervenir en él, lo que significa que de todos modos ya ocurre la diferenciación funcional de la economía. Este es también el sentido adicional y más profundo de la exigencia de Quesnay relativa a basar la moral en la física.

Se puede seguir el desarrollo posterior de este tipo de teorías del sistema en el sistema de Quesnay a través de Smith y Ricardo (reemplazando principalmente aquí la diferencia entre ricos/pobres por la diferencia entre capital/trabajo) hasta llegar a Keynes y Friedman. Estas teorías continuadoras están arrojadas sin embargo además a una evolución de ideas relativamente independiente, la cual corre más rápido que la evolución estructural del sistema que describen. Esto da lugar a complejas interdependencias que deben ser investigadas cuidadosamente. Uno no puede en este caso atenerse solamente a las teorías de la evolución o la revolución en la ciencia (Latsis 1976). Si se tiene que asumir que las teorías transforman su objeto cuando lo emplean, se debe contar con que ellas mismas pueden anularlo mediante publicaciones científicas. A la antigua crítica política, vigente al menos desde el siglo XVIII, relativa a la influencia de la teoría económica ${ }^{53}$ se le añade luego un problema epistemológico: la economía misma cambia debido a su descripción. La experiencia acumulada puede participar entonces en la

\footnotetext{
${ }^{50}$ Especialmente las formulaciones iniciales de Quesnay ponen en claro este carácter "autopoiético" de la economía: "Un hombre puede adquirir riqueza solo a través de la riqueza que ya posee, y por medio de las ganancias que la riqueza de los demás procuran para él" (citado según Meek 1963: 391). Otros autores contemporáneos reconocen de manera mucho más aguda que la riqueza produce pereza y que solamente la riqueza de los demás (es decir, solamente el sistema de la economía) incita a la invención, el afán y la actividad. El sistema se activa sí mismo y para que esto sea posible debe ser liberado. Véase Reimarus (1768: 41).

51 "Rompiendo el círculo" lo formula también Meek (1963: 292, 375).

${ }^{52}$ Sobre el problema de los gremios, especialmente la discusión alemana relativa a esto. Véase, por ejemplo, Schlettwein (1772-1773, vol. 2: 183 y ss.).

${ }^{53}$ Véase, por ejemplo Mably (1768) o Linguet (1770: 14 y ss.). En relación con los fisiócratas o con la economía política de su tiempo Hodgskin (1827: 263 y s.).
} 
evolución de la semántica de autodescripción, así como en la evolución del instrumental teórico del análisis científico; pero junto a esto hay siempre procedimientos científicos de comprobación y además variaciones que son tratadas sencillamente como "casualidades" en el contexto de la evolución. Para poder entender cómo funciona todo esto en conjunto y qué efectos tiene esta interacción se requeriría de una cuidadosa exploración, la que no podría evitar tomar como base a una teoría de sistemas autorreferenciales para abordar dichos temas.

El hecho que el sistema responda a su propia descripción, transforma la formulación del problema que vincula "teoría" y "praxis". Durante mucho tiempo, desde Quesnay hasta Ricardo, se trató simplemente de una sencilla traducción de la función del sistema, a saber: aumentar la prosperidad. La economía de mercado establecida en base a esto produjo el problema del desempleo y el problema del retiro -condicionado políticamente y por los sindicatos- de los costos del trabajo desde los mecanismos del mercado. La teoría que intentó reaccionar ante esto fue aquella formulada por Keynes. El problema que surgió desde allí pareció ser: cómo calcular las inversiones si es que no hay precios políticamente independientes $\mathrm{y}$, particularmente, si ya no existe un precio para el dinero (tasa de interés) políticamente independiente. Las teorías de reflexión se dinamizan, en este sentido, por su propio efecto sobre el sistema. No solo deben proporcionar un "progreso del conocimiento", sino sobre todo reaccionar al cambio de estado motivado por ellas mismas, reemplazando su formulación de problemas y probablemente también sus diferencias directrices (como por ejemplo, ricos/pobres, capital/trabajo, inversión/ consumo).

Para un análisis sociológico de esta historia teórica resulta importante tomar en cuenta, entre otras cosas, que la formación de teorías se ha trasladado a los subsistemas. Esto vale de igual modo; cuando se concibe el desarrollo como una autorreflexión del sistema económico, y también cuando se lo clasifica en la historia de las disciplinas como una teoría de los objetos del sistema de la ciencia. Ambas concepciones van, de hecho, de la mano y no pueden separarse empíricamente. La reflexión del sistema económico reemplaza, por así decir, sus soportes: en vez de religión, moral, derecho o política ahora se apoya en la ciencia y obtiene así una mayor libertad para su autodescripción ${ }^{54}$. La pregunta sociológicamente interesante es por tanto: cómo una semántica de la economía, que tiene que demostrar su valor en el nivel del subsistema funcional, se delimita frente a su historia y sobre todo frente a la reflexión transversal a toda la sociedad.

En términos generales se puede decir que las teorías de reflexión reaccionan frente a la diferenciación de la sociedad y con ello refuerzan la diferenciación de la sociedad. Ellas formulan problemas especiales para su sistema y refinan su sensibilidad de un modo que implica indiferencia frente a todo lo demás. En el caso del sistema económico, el punto central parece ser que las nuevas teorías de reflexión y los análisis científicos de la economía tienen que tener sangre fría frente a la diferencia entre ricos y pobres, mientras que la reflexión transversal a toda la sociedad no puede hacerlo. Esta diferencia posee por un lado una dimensión histórica. Ricos y pobres fueron solamente un aspecto de la estratificación de la vieja sociedad, de manera casi idéntica a la mera necesidad de orden. Con la disolución del orden social medieval esta diferencia quedó en cierta medida sin utilidad. Para los fisiócratas constituía una necesidad de la naturaleza y estaba ubicada por tanto fuera de la sociedad, fuera de las autorregulaciones de las relaciones económicas. Poco más tarde, el acercamiento entre la terminología social y económica condujo a la tesis (presupuesta ya por Hegel) que la sociedad como sistema produce la necesidad de esta diferencia. En la teoría de la sociedad dicha diferencia sigue siendo central, frente a la cual la teoría económica debe tomar distancia (tiene que hacer frente,

\footnotetext{
${ }^{54}$ Acerca del proceso paralelo en el sistema educativo, el cual se basa fundamentalmente en un uso inflacionario de la filosofía kantiana, véase Luhmann (1981f; Luhmann \& Schorr 1979).
} 
por supuesto, a problemas de distribución o de poder de compra, a la orientación al consumo o al tamaño del mercado, etc.; pero no puede de ninguna manera considerar la unidad de la economía - ¡su tema!- como diferencia entre ricos y pobres). Hegel y Marx tuvieron en mente esta preocupación, la cual condujo especialmente a Marx a elaborar la "crítica de la economía política" como una teoría del capitalismo para los capitalistas.

Esto no constituye por supuesto una solución, pues no son los capitalistas quienes escriben esta teoría, sino que esta se corresponde exactamente con las condiciones y consecuencias de la diferenciación funcional en el ámbito de la economía (y por lo tanto su crítica debe ser complementada mediante las críticas correspondientes en todos los otros sistemas funcionales). La teoría de sistemas autopoiéticos que anteriormente hemos tratado de aplicar tiene, por su parte, ciertas ventajas. Puede recibir estímulos de investigaciones muy generales (y al mismo tiempo empíricas). Es aplicable (con diferentes operacionalizaciones) al propio sistema de la sociedad y a los sistemas funcionalmente diferenciados de la sociedad. Ella hace precisamente esta diferencia como parte de su problema, vale decir la improbabilidad evolutiva y los riesgos de la diferenciación de los subsistemas autopoiéticos. Sin embargo, probablemente sea demasiado compleja y demasiado esotérica para el uso diario en la comunicación social. No se ubica a sí misma en las inmediaciones del espíritu mundial (lo que le acarreó a Hegel la dificultad de no poder decir quién realmente escribía su teoría). No se ubica tampoco cerca del crudo impulso de arrebatarle sus posesiones a la gente acomodada. ¿Pero se debiera suponer que tales maniobras son necesarias para aportar a la autorreflexión en el proceso de comunicación de la sociedad?, ¿debe haber entonces razón y violencia? RM

\section{Bibliografía}

Adorno, T. W. (Ed.) (1969). Spätkapitalismus oder Industriegesellschaft? Stuttgart: Enke.

Appleby, J. (1976). Ideology and Theory: The Tension Between Political and Economic Liberalism in Seventeenth Century England. American Historical Review, 81(3), 499515.

Baum, R. C. (1976). Introduction Part IV: Generalized Media in Action. En J. J. Loubser et al. (Eds.), Explorations in General Theory in Social Science: Essays in Honor of Talcott Parsons, vol. 2 (pp. 448-468). New York: The Free Press.

Baum, R. C. (1976a) Communication and Media. En J. J. Loubser et al. (Eds.), Explorations in General Theory in Social Science: Essays in Honor of Talcott Parsons, vol. 2 (pp. 533- 556). New York: The Free Press.

Belshaw, C. S. (1965). Traditional Exchange and Modern Markets. Englewood Cliffs N.J; Prentice- Hall..

Boesnier de l'Orme (1775). L'esprit du gouvernement économique. Paris.

Chalk, A. F. (1951). Natural Law and the Rise of Economic Individualism in England. Journal of Political Economy, 59(4), 332- 337.

Desmarests de Saint- Sorlin, J. (1661). Les Délices de l'esprit. Paris.

Ehrlich, H. B. (1955). British Mercantilist Theories of Profit. The American Journal of Economics and Sociology, 14(4), 377- 386.

Elster, J. (1981). Logik und Gesellschaft: Widersprüche und mögliche Welten. Frankfurt a.M.: Suhrkamp.

Emery, F. E. \& Trist, E. L. (1965). The Causal Texture of Organizational Environments. Human Relations, 18(1), 21- 32.

Grice- Hutchinson, M. (1978). Early Economic Thought in Spain 1170-1740. London: Allen and Unwin.

Gunn, J. A. W. (1969). Politics and the Public Interest in the Seventeenth Century. London: Routledge \& Kegan Paul

Habermas, J. (1980). Handlung und System - Bemerkungen zu Parsons' Medientheorie. En W. Schluchter (Ed.), Verhalten, Handeln und System: Talcott Parsons' Beitrag zur Entwicklung der Sozialwissenschaften (pp. 68- 105). Frankfurt a.M.: Suhrkamp.

Habermas, J. (1981). Theorie kommunikativen Handelns. Frankfurt a.M.: Suhrkamp. 
Habermas, J. \& Luhmann, N. (1971). Theorie der Gesellschaft oder Sozialtechnologie. Frankfurt a.M.: Suhrkamp.

Hegel, G. F. W. (1983). Philosophie des Rechts: Die Vorlesung von 1819/20 in einer Nachschrift (D. Henrich, Ed.). Frankfurt a.M.: Suhrkamp.

Hodgskin, T. (1827). Populär Political Economy. London: Charles Tait.

Kornai, J. (1971). Anti-Equilibrium: On Economic Systems Theory and the Tasks of Research. Amsterdam: North- Holland Publishing Company.

Krauth, W.-H. (1982). Marckht-Ordnung - Oeconomia-Landwürthschaft: Studien zur wirtschaftlichen Semantik in Deutschland zwischen dem 13. und 17. Jahrhundert. Tesis doctoral. Berlin.

Latsis, S. J. (1976). Method and Appraisal in Economics. Cambridge: Cambridge University Press.

Linguet, S.- H.- N. (1770). Lettres sur la Theorie des loix civiles. Amsterdam.

Luhmann, N. (1970). Wirtschaft als soziales System. En N. Luhmann, Soziologische Aufklärung 1: Aufsätze zur Theorie sozialer Systeme (pp. 204-231). Opladen/Köln: Westdeutscher Verlag.

Luhmann, N. (1972). Knappheit, Geld und die bürgerliche Gesellschaft. Jahrbuch für Sozialwissenschaften, 23(2), 186- 210.

Luhmann, N. (1975). Macht. Stuttgart: Enke.

Luhmann, N. (1975a). Einführende Bemerkungen zu einer Theorie symbolisch generalisierter Kommunikationsmedien. En N. Luhmann, Soziologische Aufklärung 2: Aufsätze zur Theorie der Gesellschaft (pp. 170-192). Opladen: Westdeutscher Verlag.

Luhmann, N. (1975b). Systemtheorie, Evolutionstheorie und Kommunikationstheorie. En N. Luhmann, Soziologische Aufklärung 2: Aufsätze zur Theorie der Gesellschaft (pp. 193- 203). Opladen: Westdeutscher Verlag.

Luhmann, N. (1977). Funktion der Religion. Frankfurt a.M.: Suhrkamp.

Luhmann, N. (1980). Frühneuzeitliche Anthropologie: Theorietechnische Lösungen für ein Evolutionsproblem der Gesellschaft. En N. Luhmann, Gesellschaftsstruktur und Semantik: Studien zur Wissenssoziologie der modernen Gesellschaft, vol. 1 (pp. 162234). Frankfurt a.M.: Suhrkamp.

Luhmann, N. (1981). Zur Funktion der »subjektiven Rechte«. En N. Luhmann, Ausdifferenzierung des Rechts: Beiträge zur Rechtssoziologie und Rechtstheorie (pp. 360- 373). Frankfurt a.M.: Suhrkamp.

Luhmann, N. (1981a). Subjektive Rechte: Zum Umbau des Rechtsbewusstseins für die moderne Gesellschaft. En N. Luhmann, Gesellschaftsstruktur und Semantik. Studien zur Wissenssoziologie der modernen Gesellschaft, vol. 2 (pp. 45-104). Frankfurt a.M.: Suhrkamp.

Luhmann, N. (1981b). Theoretische und praktische Probleme der anwendungsbezogenen Sozialwissenschaften. En N. Luhmann, Soziologische Aufklärung 3: Soziales System, Gesellschaft, Organisation (pp. 321-334). Opladen: Westdeutscher Verlag.

Luhmann, N. (1981c). Politische Theorie im Wohlfahrtsstaat. München: Günter Olzog Verlag.

Luhmann, N. (1981d). Die Unwahrscheinlichkeit der Kommunikation. En N. Luhmann, Soziologische Aufklärung 3: Soziales System, Gesellschaft, Organisation (pp. 25-34). Opladen: Westdeutscher Verlag.

Luhmann, N. (1981e). Die Ausdifferenzierung von Erkenntnisgewinn: Zur Genese von Wissenschaft. En N. Stehr \& V. Meja (Eds.), Wissenssoziologie. Sonderheft 22 (1980) der Kölner Zeitschrift für Soziologie und Sozialpsychologie (pp. 101-139). Opladen: Westdeutscher Verlag.

Luhmann, N. (1981f.). Theoriesubstitution in der Erziehungswissenschaft: Von der Philanthropie zum Neuhumanismus. En N. Luhmann, Gesellschaftsstruktur und Semantik. Studien zur Wissenssoziologie der modernen Gesellschaft, vol. 2 (pp. 105194). Frankfurt a.M.: Suhrkamp.

Luhmann, N. (1982). Liebe als Passion: Zur Codierung von Intimität. Frankfurt a.M.: Suhrkamp. 
Luhmann, N. (1983). Das sind Preise. Soziale Welt, 34(2),153- 170.

Luhmann, N. (1983a). Zum Begriff der sozialen Klasse. Manuscrito. [Publicado dos años más tarde: Luhmann, N. (1985). Zum Begriff der sozialen Klasse. En N. Luhmann (Ed.), Soziale Differenzierung. Zur Geschichte einer Idee (pp. 119-162). Opladen: Westdeutscher Verlag.].

Luhmann, N. (1983b). Die Einheit des Rechtssystems. Rechtstheorie, 14(2),129- 154.

Luhmann, N. (1983c). Bürgerliche Rechtssoziologie: Eine Theorie des 18. Jahrhunderts. Archiv für Rechts- und Sozialphilosophie, 69(4), 431- 445.

Luhmann, N. \& Schorr, K. E. (1979) Reflexionsprobleme im Erziehungssystem. Stuttgart: Klett- Cotta

Mably, Gabriel Bonnot, Abbé de (1768). Doutes Proposés aux Philosophes Économistes, sur l'ordre naturel et essentiell des sociétés politiques. Den Haag \& Paris.

Mandeville, B. (1924). The Fable of the Bees: Or Privates Vices Publick Benefits. Citado según la edición de F. B. Kaye. Oxford.

Maturana, H. (1981). Autopoiesis. En M. Zeleny (Ed.), Autopoiesis: A Theory of Living Organization (pp. 21- 35). New York: North Holland.

Maturana, H. (1982). Erkennen: Die Organisation und Verkörperung von Wirklichkeit: Ausgewählte Arbeiten zur biologischen Epistemologie. Braunschweig: Friedr. Vieweg \& Sohn.

Meek, R. L. (1963). The Interpretation of Physiocracy. En R. L. Meek, The Economics of Physiocracy: Essays and Translations. Cambridge, Mass.: Harvard University Press.

Montias, J. M. (1976). The Structure of Economic Systems. New Haven: Yale University Press.

Morellet, Abbé (1980) Prospectus d'un nouveau Dictionnaire du Commerce. Paris 1769. Reimpreso en München.

Parsons, T. (1980). Zur Theorie der sozialen Interaktionsmedien (S. Jensen, Ed.). Opladen: Westdeutscher Verlag.

Polanyi, K. (1978). The Great Transformation (1944). Citado según la traducción alemana. Frankfurt a,M.: Suhrkamp.

Rees, A. (1968). Economics. En, International Encyclopedia of the Social Sciences, vol. 4 (pp. 472-485). New York: Macmillan.

Reimarus, J. A. H. (1768). Handlungsgrundsätze zur wahren Aufnahme der Länder und zur Beförderung der Glückseligkeit ihrer Einwohner, aus der Natur und Geschichte untersucht. Cosmopolis: Hamburg.

Roover, R. de. (1958). The Concept of Just Price: Theory and Economic Policy. Journal of Economic History, 18(4), 418- 434.

Schlettwein, J. A., (1772-1773). Die wichtigste Angelegenheit für das ganze Publicum: oder die natürliche Ord-nung in der Politik überhaupt. 2 vol. Carlsruhe.

Schlettwein, J. A. (1779). Grundfeste der Staaten oder die politische Ökonomie. Gießen.

Senac de Meilhan (1787) Considerations sur l'esprit et les mceurs. London.

Thurnwald, R. (1936). Gegenseitigkeit im Aufbau und Funktionieren der Gesellungen und deren Institutionen. En, Festgabe für Ferdinand Thönnies (pp. 275-297). Leipzig: Hans Buske.

Torrens, R. (1821). An Essay on the Production of Wealth. London: Longman, Hurst, Rees, Orme, and Brown

Watt, I. (1957). The Rise of the Novel: Studies in Defoe, Richardson and Fielding. London: Chatto and Windus.

White, H. C. (1981). Where do Markets Come From. American Journal of Sociology, 87(3), 517- 547.

Whitehead, A. N. (1979). Prozeß und Realität: Entwurf einer Kosmologie. Frankfurt a.M: Suhrkamp. 


\section{Sobre el autor}

Niklas Luhmann (1927-1998) es considerado uno de los sociólogos más importantes del último siglo. Su mayor aporte radica en la profundización de la teoría de sistemas en el ámbito de la sociología y el desarrollo de una teoría de la sociedad de pretensiones universalistas. Desarrolló gran parte de su carrera en la universidad de Bielefeld, Alemania, desde 1968 hasta su retiro en 1993, siendo el primer profesor que tuvo dicha universidad. Falleció el 6 de Noviembre de 1998. Niklas Luhmann escribió durante su vida más de cincuenta libros y cientos de artículos, por lo que es muy difícil una visión sucinta de su obra. Sin embargo podemos destacar entre sus últimos libros traducidos al español: La sociedad de la sociedad (Herder- Universidad Iberoamericana, 2007), Organización y decisión (Herder-Universidad Iberoamericana, 2010) y El amor (Prometeo Libros, 2012).

\section{Contacto}

(Para consultas sobre su vida y obra)

Universität Bielefeld

Fakultät für Soziologie

Postfach 100131

33501, Bielefeld

Deutschland

Recibido: Abril 2013

Aceptado: Mayo 2013 